\title{
Ground and flight performances of the balloon-borne magnetic spectrometer AESOP-Lite
}

\author{
P.-S. Mangeard ${ }^{* a}$, J. Clem $^{a}$, P. Evenson ${ }^{a}$, R.P. Johnson ${ }^{b}$, B. Lucas ${ }^{a}$, S. Mechbal ${ }^{b}$, J. \\ Roth $^{a}$ \\ ${ }^{a}$ Bartol Research Institute, University of Delaware, Newark, DE 19716, USA. \\ ${ }^{b}$ Santa Cruz Institute for Particle Physics, Physics Department, University of California Santa \\ Cruz, Santa Cruz, CA 95064, USA. \\ E-mail: mangeard@udel.edu, clem@bartol. udel.edu, evenson@udel.edu, \\ rjohnson@ucsc.edu, lucasb@udel . edu smechbal@ucsc .edu, \\ roth@bartol.udel.edu
}

The Anti-Electron Sub-Orbital Payload Low Energy (AESOP-Lite) is designed to explore the origin of the negative spectral index in the cosmic ray electron spectrum below $100 \mathrm{MeV}$ through a series of balloon flights. The original entry telescope from the Classic LEE (Low Electron Energy) instrument has been directly integrated in AESOP-Lite. The instrument utilizes a gas Cherenkov and magnetic spectrometer configuration to identify particle type and determine the energy. The first flight took place May 15-21, 2018 from Kiruna, Sweden accumulating roughly 130 hours of exposure above 130,000 feet altitude before landing on Ellesmere Island, Canada. In this paper, we report on its design, calibration and performances. This includes the analyses of ground data taken during the integration of the detector before the flight. The observed muon charge separation from ground runs is discussed and compared to the expected performance of the spectrometer. The energy resolution from track reconstruction algorithms and the energydependent geometry factor are tested with Monte Carlo simulations. Finally, we present a brief overview of the 2018 flight and preliminary results of the detector performances in flight.

36th International Cosmic Ray Conference -ICRC2019-

July 24th - August 1st, 2019

Madison, WI, U.S.A.

\footnotetext{
*Speaker.
} 


\section{Introduction}

We introduce AESOP-Lite (The Anti-Electron Sub-Orbital Payload), a balloon-borne magnetic spectrometer designed to measure primary electron and positron cosmic rays from $20 \mathrm{MeV}$ to $300 \mathrm{MeV}$. The main science goal of the mission is to study the effects of solar modulation at $1 \mathrm{AU}$ in this scarcely-explored energy regime. AESOP-Lite has successfully completed its first mission in May 2018, on a 5-day flight between Esrange, Sweden (66.89'N) and Ellesmere Island, Canada (78.40'N), on a NASA 40 million cubic feet, zero pressure, long duration balloon. AESOP-Lite can detect particles in an energy range that both PAMELA [1,2] and AMS-02 [3] are not sensitive to, while also overlapping in sensitivity with the Voyager spacecrafts, which are now probing the unmodulated Local Interstellar Spectrum outside the heliopause [4]. This paper will give an overview of the instrument, its acceptance, resolution and performance.

\section{AESOP-Lite instrument}

Our instrument is the successor of the LEE (Low Energy Electrons) payload [5], which retired after 23 successful flights, providing important measurements of low-energy electrons. LEE observations have highlighted the mysterious origin of the turn-up in the electron spectrum below 100 $\mathrm{MeV}$, and the discrepancies between observations in the inner and outer heliosphere. However, no further conclusions could be reached until simultaneous measurements of electrons and positrons were made. To this effect, the instrument has been modified by replacing the original calorimeter with a magnetic spectrometer, making charge-sign distinction possible. The original entry telescope has been kept: it consists of three plastic scintillators and a Cherenkov gas detector. Figure 1 shows a schematic cross-section of the instrument. The main consideration in designing the pay-

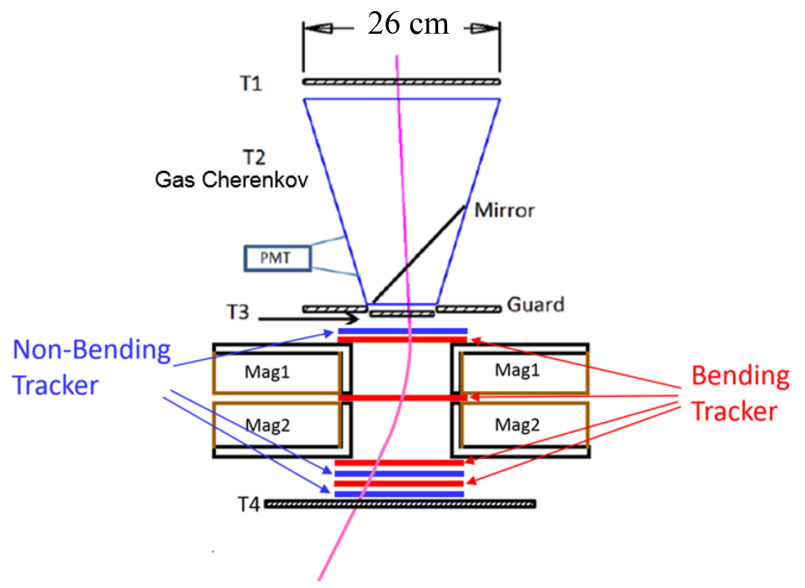

Figure 1: Diagram of the AESOP-Lite instrument. The spectrometer consists of 7 planes of silicon strip detectors

load is to reduce the weight required to reach higher altitudes on a balloon in order to minimize the contribution of secondary cosmic rays produced in the residual atmosphere (below a few $\mathrm{g} \mathrm{cm}^{-2}$ ). In addition, the geometry factor must be large enough such that good statistical accuracy can be reached, despite the low flux of primary electrons. 


\subsection{Entry telescope}

The entry telescope consists of three NE 102 A plastic scintillators (T1, T3 and Guard) and a gas Cherenkov detector (T2). T1 and T3 both identify singly charged relativistic particles, while T2 serves as a hadron discriminator. The Cherenkov counter is filled with $C_{3} F_{8}$ gas to an absolute pressure of $1.8 \mathrm{~atm}$, rejecting all particles with $\gamma=\mathrm{E} / \mathrm{mc}^{2} \leq 15.7$ (corresponding to an $e^{+} / e^{-}$ energy of approximately $8 \mathrm{MeV}$ ). Furthermore, T2 only accepts downward-moving particles, thus removing upward-going splash albedo present in the atmosphere. The T1-T2-T3 coincident signal is used in flight as the main trigger for the tracker system and also defines the geometric acceptance of the instrument. The guard counter $(\mathrm{G})$, strictly used offline in anti-coincidence, serves to flag particles produced by showers inside the apparatus, while the plastic scintillator T4, placed at the very bottom, selects particles that have completely penetrated the instrument. Since the pulse height analyzers (PHA) and front-end electronics of each counter-photomultiplier tube (PMT) system have been used in past LEE flights, we are hence provided with an ability to cross-calibrate the absolute electron fluxes with previous measurements.
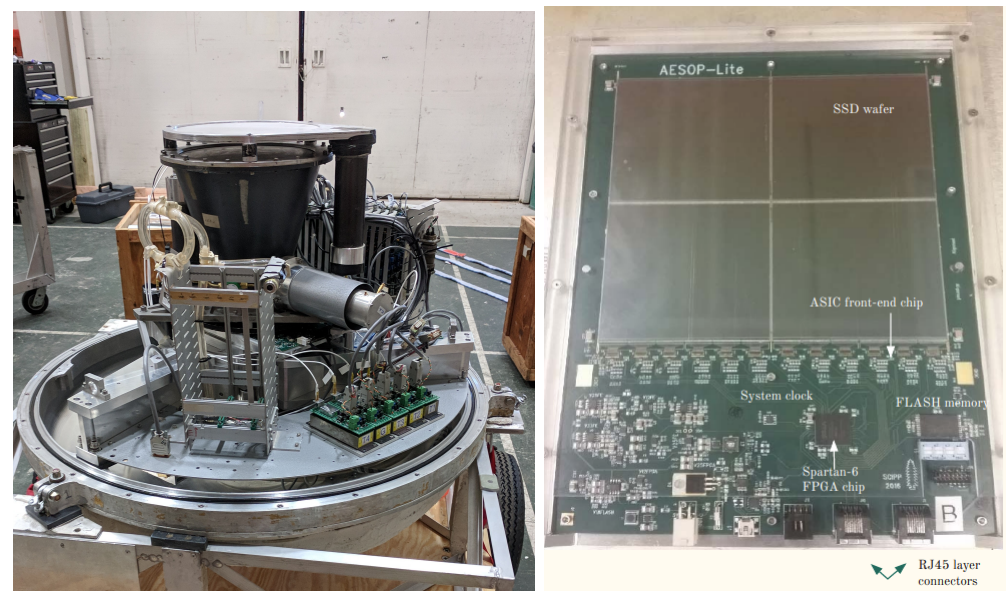

Figure 2: (Left) Photograph of the detector. (Right) Photograph of a tracker module. The 4 SSD wafers are vertically wire-bonded, all 768 channels are connected to 12 front-end ASIC chips.

\subsection{Spectrometer}

The tracking system consists of seven planes of silicon strip detectors (SSD) and a Halbach ring dipole magnet. The average field is $0.3 \mathrm{~T}$, though its known non-uniformity must be accounted for. The SSDs are arranged in an xy-configuration, with 4 layers in the bending plane to measure the particle deflection, and 3 layers to view their trajectory in the non-bending plane. The magnet design allows the placement of a tracker in the bending-view at the center of the field. The silicon wafers were custom designed and manufactured for the Large Area Telescope (LAT) of the NASA Fermi mission [6]. Each SSD is a $8.95 \times 8.95 \mathrm{~cm}^{2}, 400 \mu \mathrm{m}$ thick single-sided detector, with strip pitch $228 \mu \mathrm{m}$ and spatial resolution detector $66 \mu \mathrm{m}(228 / \sqrt{12})$. The right panel of Figure 2 shows a picture of one of the instrument's tracker module. All channels are wire-bonded to 12 ASIC (Application Specific Integrated Circuit) chips [7], which set a single common threshold with the possibility to mask any set of channels from the trigger output and/or the data flow. In addition to the data output, an asynchronous OR of all channels is provided by each chip for triggering. 
Threshold crossings are stored in a FIFO (First In First Out) for each channel pending a trigger decision, and then zero-suppressed hit lists are buffered for up to four events and delivered by LVDS (Low-Voltage Differential Signaling) to the data acquisition in a serial stream. The ASIC was designed and fabricated for a Proton Computed Tomography (pCT) system [8], which detects and measures more than 1 million protons/second with less than 5\% dead time, operating at a 100 MHz clock rate. However, the AESOP-Lite system operates the ASICs at $10 \mathrm{MHz}$ to save power, and for simplicity does not make use of the on-chip buffering, as is allowed by the relatively low cosmic-ray event rate.

\subsection{Online trigger system}

In flight, coincidences $\mathrm{T} 1-\mathrm{T} 2-\mathrm{T} 3$ and $\mathrm{T} 1-\mathrm{T} 2-\mathrm{T} 4$ were both used as an online trigger in flight (the "GO" signal). The tracker system self-triggers with a logical OR of two triggers: one from the bending view, the other from the non-bending, requiring in each view a coincidence of the top 3 layers. The data sit in each board until a "GO" trigger from the PMTs is received. If a "GO" signal fails to arrive within $5 \mu$ s, the data will be discarded.

\section{Simulated performances}

\subsection{Monte-Carlo simulation}

Comprehensive Monte Carlo simulations of the instrument were made using the FLUKA software $[9,10]$. The full geometry describes the entry telescope, 7 strip-separated silicon layers, and the non-uniform $5 \mathrm{~mm}$ step magnetic field map provided by the magnet manufacturer. The digitization of tracking ladder strips, as well as the existing gap between the sensor ladders, which is the source of most of the tracking inefficiency (as visible in the right panel of Figure 2), are also taken into account in the simulation. In flight configuration, the instrument sits inside a pressure vessel consisting of $2.5 \mathrm{~cm}$ of polyurethane foam coated on an aluminum shell $(2 \mathrm{~mm}$ of thickness), which is also modeled. The left panel of Figure 3 shows the geometry modeled using the Flair interactive software [11]. Electrons, positrons, muons, protons and alpha particles at various energies are injected separately in the simulation. The source beam and zenith angle distribution have been chosen to optimize computing time, while still covering the full acceptance of the instrument. We use the Monte Carlo simulations to develop a track reconstruction algorithm and estimate the geometry factor. Using these results, we construct the momentum reconstruction probability density functions (p.d.f.) used in our analysis, which include the resolution, bias, and efficiency of the reconstruction.

\subsection{Geometry factor}

Following the method outlined in [12], we use Monte Carlo simulations to determine the geometry factor of the AESOP-Lite instrument for each particle type studied and zenith angle bin. The right panel of Figure 3 shows the acceptance for electrons when imposing the flight trigger requirements: a signal in $\mathrm{T} 1, \mathrm{~T} 2$ and $\mathrm{T} 3$, and a reproduction of the internal tracker trigger. As expected, the geometry factor grows with momentum, where the deflecting effects of the magnetic field are mitigated: the acceptance is $\sim 7 \mathrm{~cm}^{2} \mathrm{sr}$ at $30 \mathrm{MeV} \mathrm{c}^{-1}$ and $\sim 14 \mathrm{~cm}^{2} \mathrm{sr}$ at $300 \mathrm{MeV} \mathrm{c}^{-1}$. 

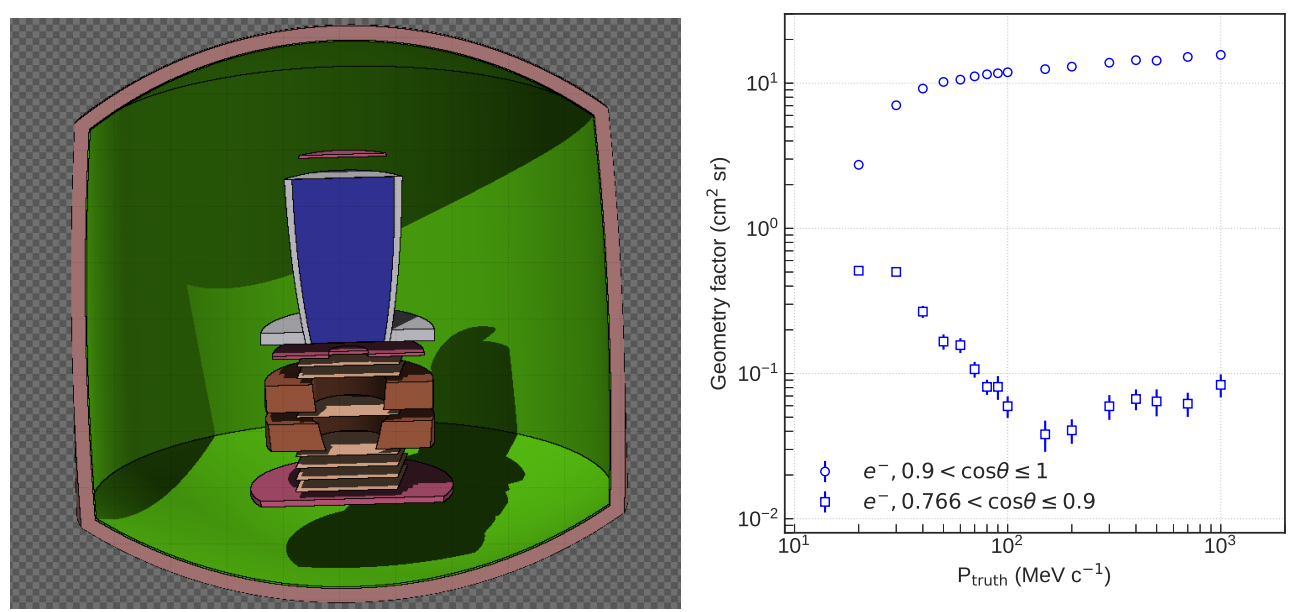

Figure 3: (Left) Model of the AESOP-Lite instrument viewed in the graphic interface Flair. (Right) Simulated post-trigger geometry factor for electrons. The calculations are made for two ranges of incident angle $\theta$.

\subsection{Track Reconstruction}

Once an event has successfully passed the selection criteria of the online trigger coincidence (for instance T1-T2-T3), it is first processed with a pattern recognition (PR) routine which selects hits that belong to a same track: $i$ ) in the non-bending view, the algorithm fits all possible lines between the top-most and bottom-most layers, and chooses the track that minimizes the $\chi^{2}$; ii) similarly in the bending view, a parabola is fit to all possible configurations of hits in the four layers of the plane and the best fit is chosen. By measuring the radius of curvature of the parabola, the deflection - and thus momentum - of the particle can be inferred. The value from the PR fit is then used to initialize the final reconstruction method. However, for this preliminary analysis, the PR routine was used as the primary reconstruction technique. A Kalman Filter [13] as well as a Runge-Kutta algorithm have been developed and are being tested. Figure 4 illustrates the parabola fit and straight line fit as seen in solid blue lines in our event display tool. The dashed lines in the bending plane panel indicate the incoming and outgoing directions of the particles assuming no scattering or interaction in the detector.

Since the distribution of the reconstructed inverse momentum - and not the momentum itself follows a normal distribution, we derive, for each reconstructed energy and bin in zenith angle, $\theta$, the $1 / p_{\text {reco }}$ distribution and fit it to a Gauss function. The reconstruction p.d.f. also contains the information of the particle's energy loss as it will have traversed the aluminum foam shell, the scintillators $\mathrm{T} 1$ and $\mathrm{T} 3$, as well as the $C_{3} F_{8}$ Cherenkov gas prior to reaching the spectrometer. The resolution, bias, and efficiency of the reconstruction are thus parametrized for 16 energies for electrons and positrons. Some examples of p.d.f. are shown in the left panel of Figure 5. The parameters of the fits are then individually and linearly interpolated to extend the knowledge to the entire energy range of the instrument. The same method is used for all particle types.

On average, we estimate the compounded energy loss to be $\sim 4 \mathrm{MeV}$, a value independent of the energy of the incident particle. The center panel in Figure 5 presents the resolution of our current reconstruction algorithm for electrons: it is about $11.5 \%$ at $30 \mathrm{MeV} \mathrm{c}^{-1}$ and $13.5 \%$ at 
Event: 125115, Non bending plane
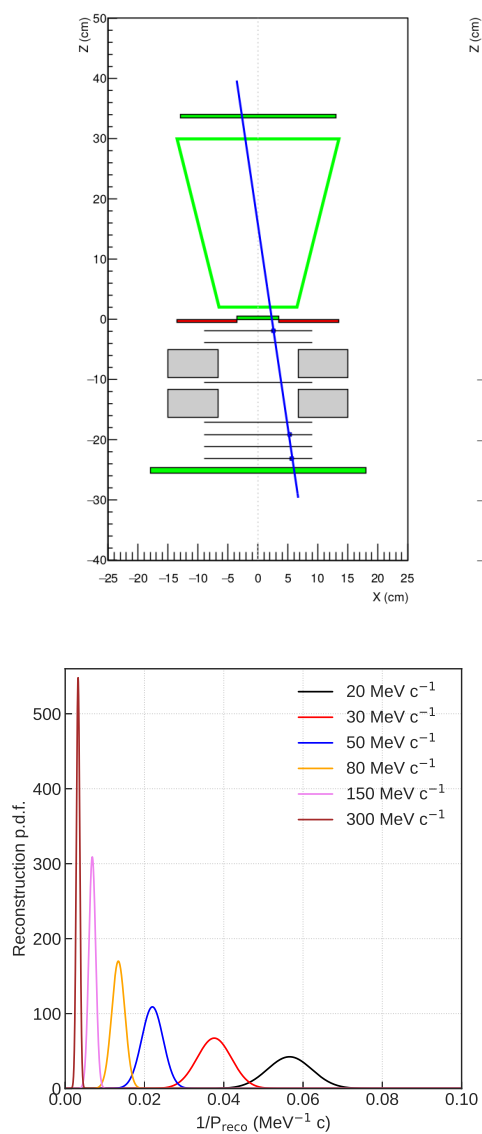

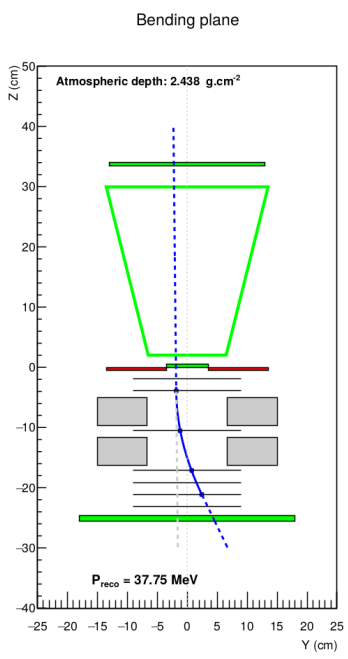

Figure 4: Event display of an electron candidate recorded during the 2018 flight. The triggers T1, T2, T3 and T4 were fired (in green) whereas no signal was seen in the guard (in red).

Figure 5: (Left) Reconstruction probability density function for the electrons (incidence: $0.9<$ $\cos \theta<1$ ). (Center) Resolution of the momentum reconstruction for electrons and (right) the associated efficiency.

$300 \mathrm{MeV} \mathrm{c}^{-1}$. The right panel in Figure 5 shows the efficiencies (post-trigger) of the selection applied to the hits occupancy in the tracker (a number of hits between 5 and 12), and the PR. The distribution shows that the highest efficiency is in our range of interest, i.e., between 30 and 100 $\mathrm{MeV} \mathrm{c}^{-1}$. Similar results are obtained for positrons.

\section{Calibration and tests at ground level}

\subsection{Ground runs}

Ground runs with different trigger configurations were performed in Esrange prior to the flight. In particular, the simple coincidence of signals in the scintillators $\mathrm{T} 1$ and $\mathrm{T} 4$ allowed us to accumulate tracks in the spectrometers without vetoing any contributions from muons with an insufficient energy to produce Cherenkov light in T2. We applied an anti-coincidence offline veto on $\mathrm{T} 2$ to select only the low energetic muons and test our ability to separate and identify positively and negatively charged particles. Figure 6 shows the measured distribution of the signed inversemomentum. We compare them with simulated distributions that combine a realistic spectrum of 
Galactic Cosmic Rays at the top of the atmosphere, atmospheric interaction shower [14], and the performances of detection presented previously in this paper. The charge separation is observed with a higher contribution of the positively charged muons as expected. There is a reasonably good agreement between calculations and observations. The measured double peaks are located at the same signed inverse-momentum as simulated. However they are slightly wider than the expected ones. This possibly indicates an overestimate of the calculated reconstruction resolution in the momentum range of several hundreds of $\mathrm{MeV} \mathrm{c}^{-1}$. Uncertainties on the simulated muon spectrum at ground level can't be neglected as a possible explanation. The peak-to-peak atmospheric muon charge ratio $\mu^{+} / \mu^{-}$is observed at $1.33 \pm 0.07$ (statistical uncertainty only).

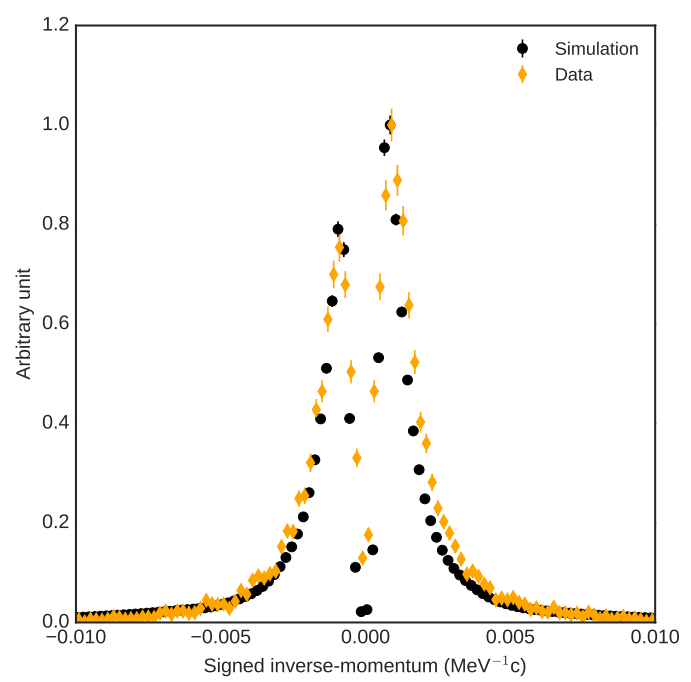

Figure 6: Ground level distribution of the signed inverse-momentum of muons as measured by AESOP-Lite in May 2019 at Esrange (orange diamonds) and estimated by simulation (black markers). Histograms are normalized to 1 at their maximum.

\subsection{Pre-flight calibration}

Calibrations of the two barometers and the PHAs of the AESOP-Lite instrument were performed in Palestine, Texas and Esrange, Sweden prior to the AESOP-Lite maiden flight. The two barometers were used in flight to record the pressure outside the shell, the accuracy of the reading being fundamental to measuring the float altitude and atmospheric overburden, an information which is crucial to our analysis method, based on the plotting of growth curves. The most sensitive region lies between 2 and $3 \mathrm{~g} \mathrm{~cm}^{-2}$ at float. Figure 7 shows the variations of one barometer readings during flight, and the corrected offset of our calibration. They are compared to the values of the CSBF barometer (Columbia Scientific Balloon Facility). For the PHA of all of our live PMT channels, a pulse generator (itself linearized using an oscilloscope) was used to check for any offsets from linearity in the pulse height reading in the data acquisition software. A $6^{\text {th }}$ order polynomial was fit to the data to calibrate the pulse heights readings. The five PHA channels were fully operational during the flight in 2018. The in-flight trigger rate in T1 is shown in the bottom panel of Figure 7.

\section{Conclusion}

The payload is a technical success with a live time of $99 \%$ during its first flight. We clearly separate electrons and positrons, and assign a good energy to each event. We have generally good 
control of the various efficiency parameters but are working to improve them. We would like to thank Matthew Collins, Forest Martinez-Mckinney, and Yang Zhou for their help in the design, construction and integration of the instrument. We thank Chris Field and the CSBF for their support during the integration period in Palestine, Texas, and for the successful balloon flight. We thank Esrange for their support during the flight campaign. This work is supported by the NASA award 80NSSC19K0746.
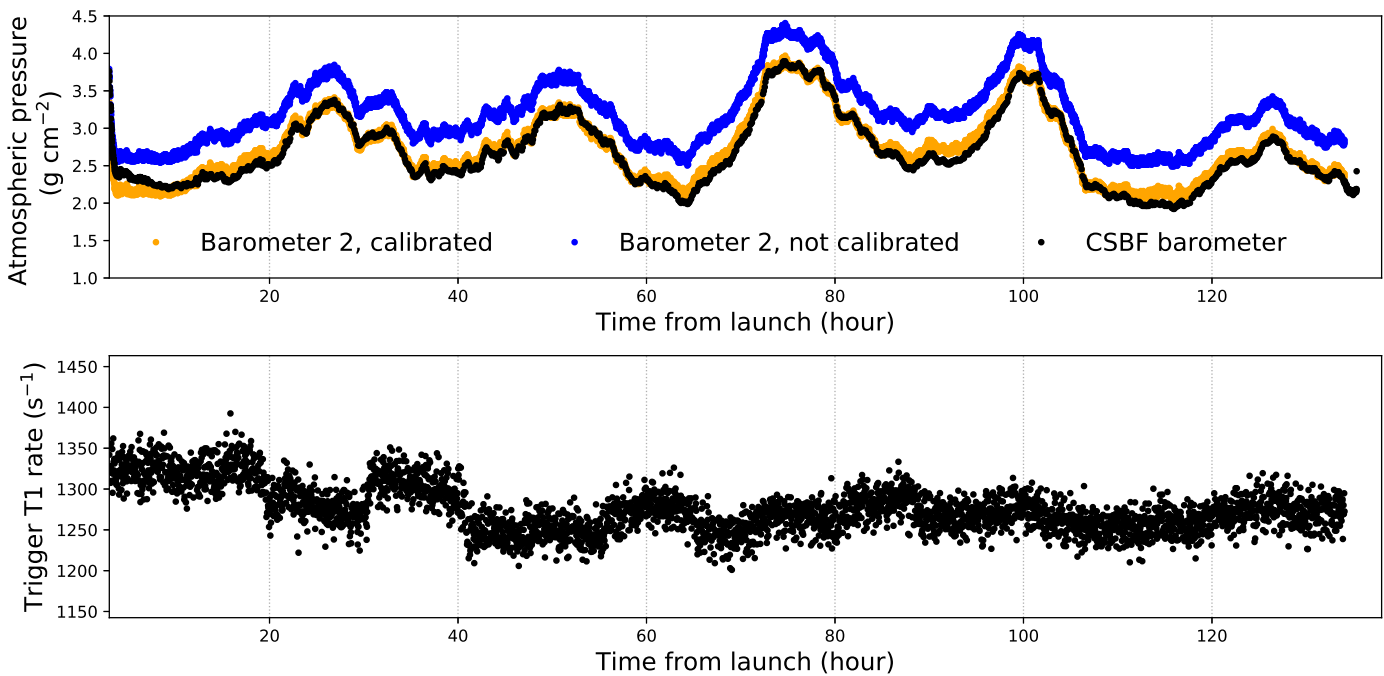

Figure 7: (Top) Time series of the calibrated and uncalibrated pressures during flight. As the sun sets below the horizon, the volume of the balloon shrinks and its altitude drops, which explains the diurnal variations. (Bottom) Trigger rate of the top scintillator T1 during the flight. Fluctuations are due to altitude changes and geomagnetic cutoff variations.

\section{References}

[1] O. Adriani et al, 2009, Nature, 458

[2] O. Adriani et al, 2015, ApJ, 810, 142

[3] M. Aguilar et al., 2013, PRL, 110

[4] A.C. Cummings et al., 2016, ApJ, 831

[5] D. Hovestadt et al., 1970, NIM, 85, 93

[6] W. Atwood et al., 2007, Astroparticle Physics, 28, 422

[7] R.P. Johnson et al, 2013, IEEE Transactions on Nuclear Science, 60, 3262

[8] R.P. Johnson et al, 2016, IEEE Transactions on Nuclear Science, 63, 52

[9] T.T. Bohlen et al., 2014, Nuclear Data Sheets 120, 211

[10] A. Ferrari et al., 2005, CERN-2005-10, INFNTC_05/11, SLAC-R-773

[11] V. Vlachoudis, 2009, Proc. Int. Conf. on Mathematics, Computational Methods \& Reactor Physics

[12] J.D. Sullivan, 1971, NIM, 95, 5

[13] R. Frühwirth, 1987, NIM, 262, 444

[14] S. Mechbal et al, 2019, PoS (ICRC2019) 1119 